Synthesis Alerts is a monthly feature to help readers of Synthesis keep abreast of new reagents, catalysts, ligands, chiral auxiliaries, and protecting groups which have appeared in the recent literature. Emphasis is placed on new developments but established reagents, catalysts etc are also covered if they are used in novel and useful reactions. In each abstract, a specific example of a transformation is given in a concise format designed to aid visual retrieval of information.

Synthesis Alerts is a personal selection by:

Elyse Bourque, Robert Chow, Jennifer Delaney, Marcel de Puit and Sukhjinder Uppal of Department of Chemistry, Leeds University, Leeds LS2 9JT, UK.

Georg Thieme Verlag does not accept responsibility for the accuracy, content, or selection of the data.

Synthesis 2001, No. 12, 2509 2001. Article Identifier: 1437-210X,E;2001,0,12,1897,1902,ftx,en;X01201SS.pdf.

(C) Georg Thieme Verlag Stuttgart · New York

ISSN 0039-7881
The journals regularly covered by the abstractors are:

Angewandte Chemie International Edition

Bulletin of the Chemical Society of Japan

Chemical Communications

Chemistry A European Journal

Chemistry Letters

Collection Czechoslovak Chemical Communications

European Journal of Organic Chemistry

Helvetica Chimica Acta

Heterocycles

Journal of the American Chemical Society

Journal of Organic Chemistry

Organic Letters

Organometallics

Perkin Transactions I

Synlett

Synthesis

Tetrahedron

Tetrahedron Asymmetry and Tetrahedron Letters

\begin{tabular}{l|l|ll}
\hline Tetrakis(triphenylphosphine)platinum(0) & Catalyst \\
\hline $\begin{array}{l}\text { The title reagent catalyses the } \\
\text { carbolithiation of alkynes. }\end{array}$ & & & \\
& & & \\
Sugoh, K.; Kuniyasu, H.; Sugae, T.; & & & \\
Ohtaka, A.; Takai, Y.; Tanaka, A.; \\
Machino, C.; Kambe, N.; Kurosawa, H.
\end{tabular}

\title{
Imidazolidinone
}

Catalyst

The title reagent promotes the asymmetric Friedel-Crafts alkylation of pyrroles with $\alpha, \beta$-unsaturated aldehydes.

Paras, N. A. MacMillan, D. W. C. J. Am. Chem. Soc. 2001, 123, 4370.

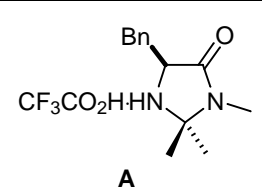

A

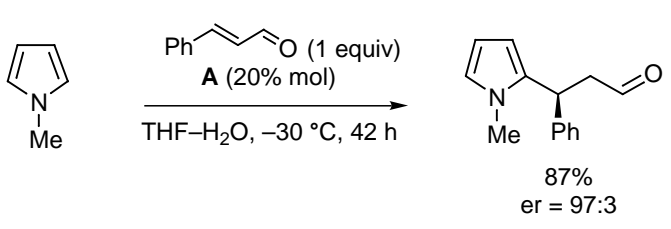

7 examples (yields 72-90\%, \%еe 87-93\%).
Bis(oxazoline) Copper(II) Complex

The title reagent promotes enantioselective and

diastereoselective Michael additions of enolsilanes to unsaturated imide derivatives.

Evans, D. A.; Scheidt, K. A.; Johnston, J. N.; Willis, M. C. J. Am. Chem. Soc. 2001, 123, 4480.

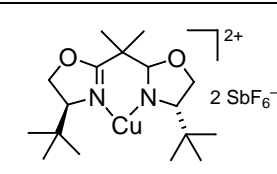

A

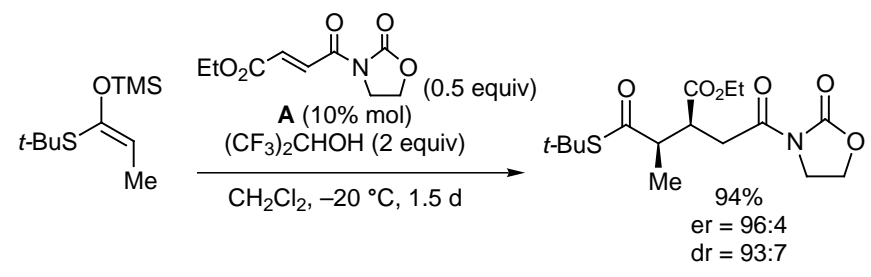

33 examples (yields 40-99\%, \%ee 40-99\%, \%de 46-98\%). 


\section{Rhodium(I) Complex} diastereoselective tandem allylic alkylation/Pauson-Khand annulation reactions.

Evans, P. A.; Robinson, J. E. J. Am. Chem. Soc. 2001, 123, 4609.
The title reagent promotes regio- and

Catalyst

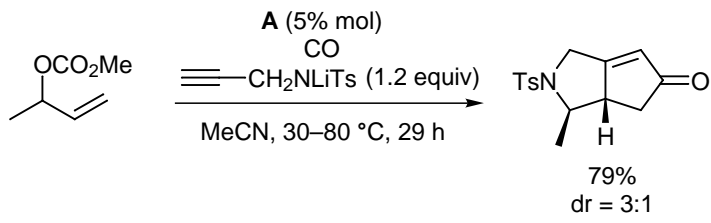

9 examples (yields $63-84 \%$, \%de 50-90\%).

\section{Triruthenium Dodecacarbonyl}

Catalyst

The title reagent promotes reductive decarboxylation of esters involving the cleavage of acyl-oxygen bonds of esters.
$[\mathrm{RhCl}(\mathrm{CO}) \mathrm{dppp}]_{2}$

A

Chatani, N.; Tatamidani, H.; le, Y.; Kakiuchi, F.; Murai, S. J. Am. Chem. Soc. 2001, 123, 4849 .

$\mathrm{Ru}_{3}(\mathrm{CO})_{12}$
$\mathrm{~A}$<smiles>CC(C(=O)OCc1ccccn1)c1ccccc1</smiles>
$\underset{\text { dioxane, } 160^{\circ} \mathrm{C}, 20 \mathrm{~h}}{\stackrel{\mathbf{A}(5 \% \mathrm{~mol})}{\mathrm{\textrm {HCOONH } _ { 4 }} \text { (3 equiv) }}}$<smiles>CCc1ccccc1</smiles>

\section{Chiral Zirconocene}

The title reagent, in the presence of water, promotes the asymmetric carboalumination of terminal alkenes, following the trimethylaluminiummediated aromatic Claisen reaction in a tandem process.

Wipf, P.; Ribe, S. Org. Lett. 2001, 3, 1503.

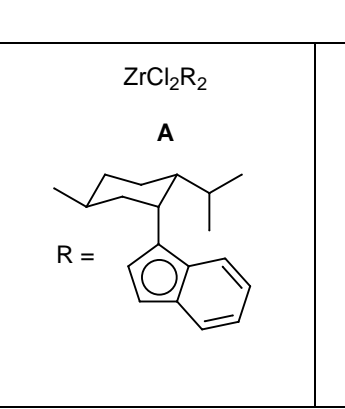

\section{Zn-Zn-Linked BINOL Complex}

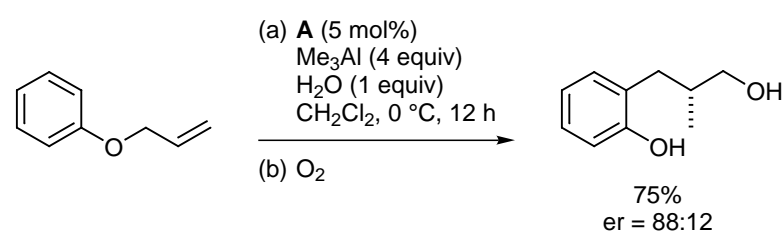

9 examples (yields 39-78\%, \%ee 60-80\%).

The title reagent promotes asymmetric aldol reactions to afford $\alpha, \beta$-dihydroxy ketones.

Kumagai, N.; Matsunaga, S.;

Yoshikawa, N.; Ohshima, T.;

Shibasaki, M. Org. Lett. 2001, 3, 1539.

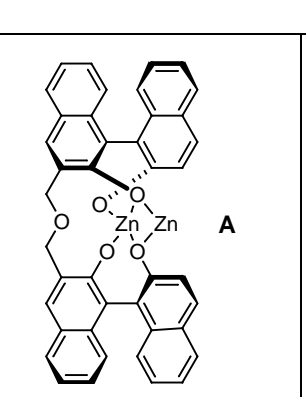<smiles>COc1ccccc1C(=O)CO</smiles><smiles>O=CCCc1ccccc1</smiles>
THF, $-20^{\circ} \mathrm{C}, 20 \mathrm{~h}$<smiles>CCCc1ccccc1</smiles>

$94 \%$

er $=96 \cdot 4$

$\mathrm{dr}=89: 1$

13 examples (yields 73-95\%, \%ee 77-99\%, \%de 20-94\%).

\section{Palladium/Carbon}

The title reagent catalyses the Suzuki cross-coupling reaction of

phenylboronic acid with aryl chlorides.

LeBlond, C. R.; Andrews, A. T.; Sun, Y.; Sowa, J. R. Jr. Org. Lett. 2001, 3 , 1555.

$\mathrm{Pd} / \mathrm{C}$
$\mathbf{A}$

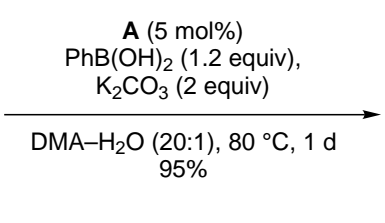<smiles>FC(F)(F)c1ccc(-c2ccccc2)cc1</smiles>

7 examples (yields $32-95 \%$ ). 
The title reagent pair catalyses the enantioselective Diels-Alder reaction of achiral 1,4-quinone monoketals with various dienes.

Breuning, M.; Corey, E. J. Org. Lett. 2001, 3, 1559.

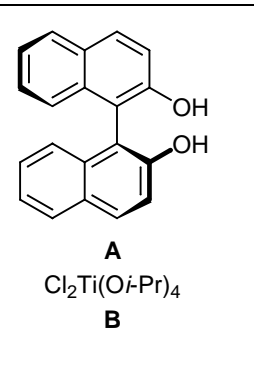

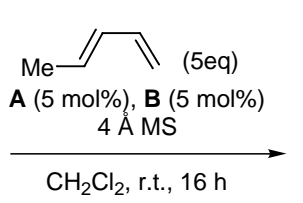

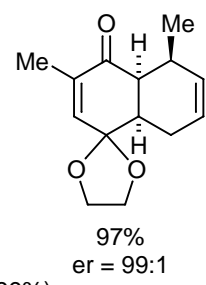

18 examples (yields 88-97\%, \%ee 87-99\%).

\begin{tabular}{l|l|l}
\hline Indium Tribromide & Catalyst \\
\hline $\begin{array}{l}\text { The title reagent catalyses the addition } \\
\text { of trimethylsilyl cyanide to } \alpha \text {-hetero- } \\
\text { substituted ketones. }\end{array}$ & & \\
Bandini, M.; Cozzi, P. G.; Melchiorre, & & \\
P.; Umani-Ronchi, A. Tetrahedron Lett. & & 10 examples (yields 27-99\%). \\
2001, 42, 3041. & & \\
\hline
\end{tabular}

\section{Molybdenum-based Chiral Catalyst}

The title reagent catalyses

enantioselective olefin metathesis.

Aeilts, S. L.; Cefalo, D. R.; Bonitatebus, P. J.; Houser, J. H.; Hoveyda, A. H.; Schrock, R. R. Angew. Chem. Int. Ed. 2001, 40, 1452.

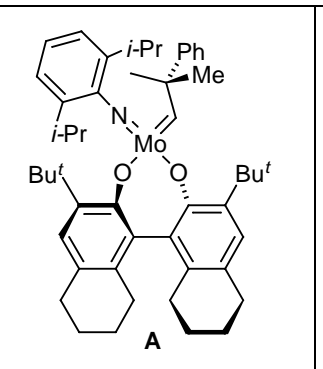

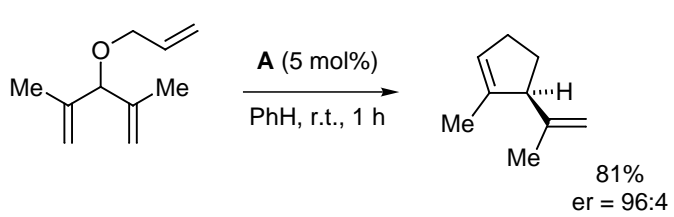

7 examples (yields 54-86\%, \%ee 92-98\%).

\section{Ruthenium Complex}

The title reagent catalyses the 3 carbon chain extension of alkynes to give enamides.

Trost, B. M.; Surivet, J.-P. Angew. Chem. Int. Ed. 2001, 40, 1468.

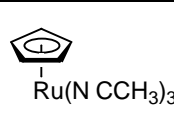

A

\section{Planar Chiral $\eta^{5}$-Cyclopentadienylrhenium(I)tricarbonyl Complex}

The title reagent catalyses enantioselective phenyl transfer to aldehydes.

Bolm, C.; Kesselgruber, M.; Hermanns, N.; Hildebrand, J. P.; Raabe, G. Angew. Chem. Int. Ed. 2001, 40, 1488.

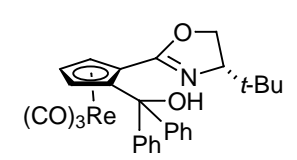

A

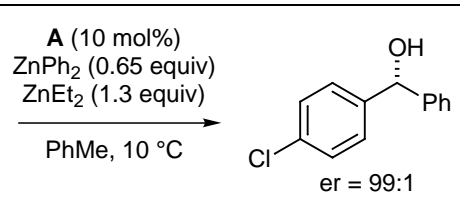

9 examples (\%ee $78-99 \%$ ). 
The title reagent catalyses the intermolecular coupling of ary bromides and oxazolidinones.

Madar, D. J.; Kopecka, H.; Pireh, D.;

Pease, J.; Pliushchev, M.; Sciotti, R. J.;

Wiedeman, P. E.; Djuric, S. W.

Tetrahedron Lett. 2001, 42, 3681.
$\mathrm{Pd}_{2}(\mathrm{dba})_{3}$

A<smiles>O=C1NC(Cc2ccccc2)CO1</smiles>

$\mathrm{Ph}$

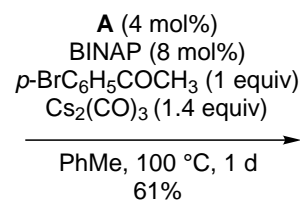<smiles>CC(=O)c1ccc(N2C(=O)OCC2Cc2ccccc2)cc1</smiles>

9 examples (yields 15-77\%).

\section{2-(2-Anisyl)-bicyclo[2.2.2]octane-2,6-diol (BODOL)}

The title ligand is employed in the titanium-catalysed asymmetric reduction of ketones with catecholborane.

Phosphoramidate

The title reagent promotes the formation of homochiral zinc enolates in a tandem enantioselective conjugate addition.

Alexakis, A.; Trevitt, G. P.;

Bernardinelli, G. J. Am. Chem. Soc. 2001, 123, 4358.

Sarvary, I.; Almqvist, F.; Frejd, T. Sarvary, I.; Almqvist, F.; Frejd,
Chem.-Eur. J. 2001, 7, 2158.

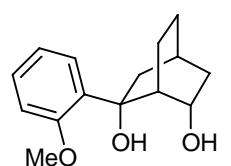

A<smiles>CC(=O)c1ccccc1</smiles>
A $(10 \mathrm{~mol} \%)$
$\left[\begin{array}{c}{\left[\mathrm{Ti}(\mathrm{O} i-\mathrm{Pr})_{4}\right](10 \mathrm{~mol} \%)} \\ \text { catecholborane }(1.5 \text { equiv })\end{array}\right.$
$\underset{\mathrm{THF},}{\mathrm{C}}-20^{\circ} \mathrm{C}, 1 \mathrm{~d}$

$\mathrm{THF},-20{ }^{\circ} \mathrm{C}, 1 \mathrm{~d}$

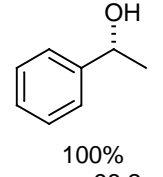

er $=98: 2$

15 examples (yields $50-100 \%$, \%ee 48-98\%).

\section{1,5-Diazadecalin}

The title ligand is employed in the copper-catalysed enantioselective oxidative biaryl coupling of substituted 2-naphthol derivatives.

Li, X.; Yang, J.; Kozlowski, M. C. Org. Lett. 2001, 3, 1137.

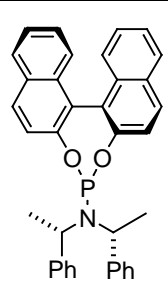

(a) A (1 mol\%)

$\mathrm{Et}_{2} \mathrm{Zn}$ (1.2 equiv)

$\mathrm{Cu}(\mathrm{OTf})_{2}(0.5 \mathrm{~mol} \%)$

$\mathrm{CH}_{2} \mathrm{Cl}_{2},-20^{\circ} \mathrm{C}, 15 \mathrm{~min}$

(b) $(\mathrm{MeO})_{3} \mathrm{CH}$ ( 1.5 equiv) $\mathrm{BF}_{3} \cdot \mathrm{OEt}_{2}$ (1.5 equiv) $\mathrm{CH}_{2} \mathrm{Cl}_{2},-20$ to $0^{\circ} \mathrm{C}, 2 \mathrm{~h}$ $66 \%$

6 examples (yields 54-66\%). copper-catalysed asymmetric conjugate reduction of $\beta$-substituted enones for the synthesis of

enantiomerically enriched 2,3-

Yun, J.; Buchwald, S. L. Org. Lett. 2001, 3, 1129.

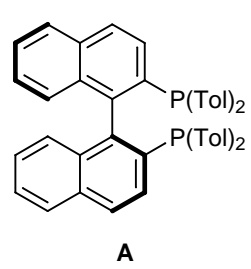

(a) A (5 mol\%), $\mathrm{CuCl}$ (5 mol\%) $\mathrm{NaOt}$-Bu (5 mol\%) $\mathrm{Ph}_{2} \mathrm{SiH}_{2}$ ( 0.5 equiv) PhMe, $0^{\circ} \mathrm{C}$

(b) TBAT (1.2 equiv), $\mathrm{BnBr}$ (2 equiv) $\mathrm{CH}_{2} \mathrm{Cl}_{2}-\mathrm{PhMe}$ (1:1), r.t., $1 \mathrm{~d}$

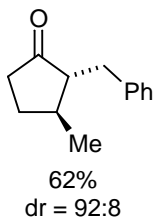

8 examples (yields $42-67 \%$, \%de $80-94 \%$ ). 
The title ligand is applied in the coppercatalysed asymmetric allylic substitution of di- and trisubstituted alkenes.

Luchaco-Cullis, C. A.; Mizutani, H.; Murphy, K. E.; Hoveyda, A. H. Angew. Chem. Int. Ed. 2001, 40, 1456.

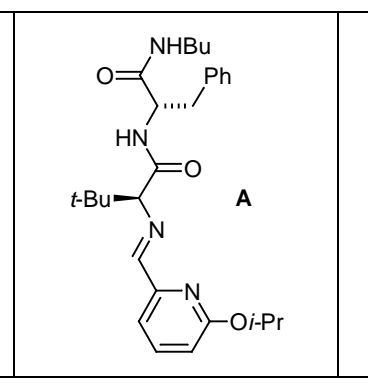

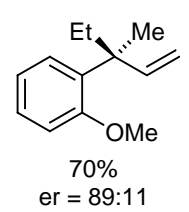

er $=89: 11$

6 examples (yields 34-96\%, \%ee 66-82\%).

\section{1,3-Dimethyl-5-[(dimethylamino)methylene]-2,4,6-(1H,3H,5H)-trioxopyrimidine}

The title reagent is used to protect aminosugars and other primary amides.

Dekany, G.; Bornaghi, L.;

Papageorgiou, J.; Taylor, S.

Tetrahedron Lett. 2001, 42, 3129

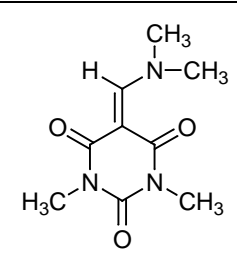

A

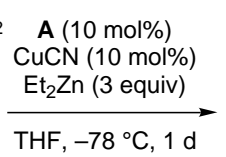

4-Isopropyl-3-(methylthiomethyl)-5,5-diphenyloxazolidin-2-one

The lithiated derivative of $A$ is used as a chiral formyl anion equivalent for the preparations of 1,2-diols, 2-amino alcohols, 2-hydroxy esters, and 4hydroxy-2-alkenoates.

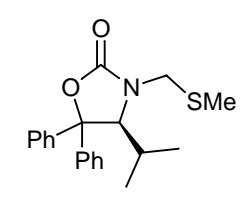

A
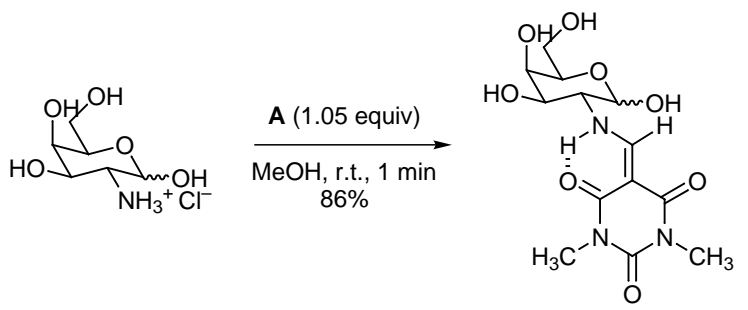

2 examples (yields $86-90 \%$ ).

\section{Triethylboron}

The title reagent plays a dual role as radical initiator and in situ derivitisation reagent in hydrogen transfer reactions involving acyclic 1,2- and 1,3-diols.

Bouvier, J.-P.; Jung, G.; Liu, Z.; Gurérin, B.; Guindon, Y. Org. Lett. 2001, 3, 1391.
$\mathrm{Et}_{3} \mathrm{~B}$

A

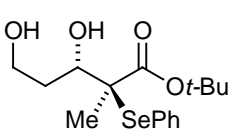

(a) $\mathbf{A}$ (1.3 equiv) $\mathrm{CH}_{2} \mathrm{Cl}_{2}, 0^{\circ} \mathrm{C}$

$\overrightarrow{\text { (b) } \mathrm{Bu}_{3} \mathrm{SnH} \text { (2 equiv) }}$

4 examples (yields $78-90 \%$, \%de 20-91\%). 


\section{Phenylsilane/Dibutyltin Chloride}

The title reagent pair promotes the direct reductive amination of aldehydes and ketones.

Apodaca, R.; Xiao, W. Org. Lett. 2001, 3, 1745.

\begin{tabular}{l|l}
$\mathrm{PhSiH}_{3}$ \\
$\mathrm{Bu}_{2} \mathrm{SnCl}_{2}$ \\
$\mathbf{B}$
\end{tabular}

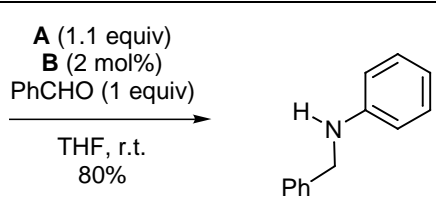

20 examples (yields 13-91\%).

\section{2-Chloro-4,6-dimethoxy[1,3,5]triazine}

Reagent

The title reagent converts carboxylic acids into activated esters under mild conditions, which are subsequently reacted with a Grignard/Cul reagent to give the corresponding ketones.

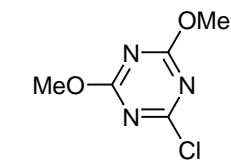

A

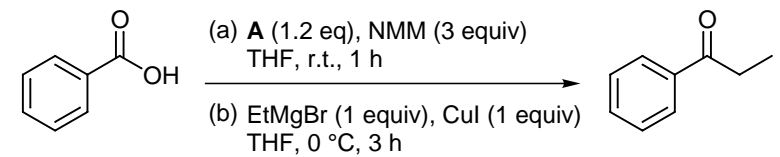
$\mathrm{THF}, 0^{\circ} \mathrm{C}, 3 \mathrm{~h}$ $98 \%$

De Luca, L.; Giacomelli, G.;

Porcheddu, A. Org. Lett. 2001, 3, 1519.

10 examples (yields $48,90-98 \%$ ) are reported.

\begin{tabular}{l}
\hline Samarium lodide \\
$\begin{array}{l}\text { The title reagent is used as a reducing } \\
\text { agent in the selective reduction of } \\
\text { conjugated } \Delta^{2} \text {-isoxazolines, which } \\
\text { after hydrolysis with } \mathrm{B}(\mathrm{OH})_{3} \text {, gives the } \\
\text { corresponding unsaturated } \beta \text {-hydroxy } \\
\text { ketones. }\end{array}$ \\
$\begin{array}{ll}\text { Bode, J. W.; Carreira, E. M. Org. Lett. } \\
\text { 2001, 3, 1587. }\end{array}$ \\
\hline
\end{tabular}

Polymethylhydrosiloxane (PMHS)-Zinc Chloride/Tetrakis(triphenylphosphine)palladium(0)

The title reagent pair is used for the selective cleavage of allyl ethers, amines and esters.

Chandrasekhar, S.; Raji, ReddyC.; Jagadeeshwar Rao, RaoR. Tetrahedron 2001, 57, 3435.

PMHS- $\mathrm{ZnCl}_{2}$
$\mathrm{Pd}\left(\mathrm{PPh}_{3}\right)_{4}$
B

\section{Tetrabutylammonium Butyldifluorodimethylsilicate}

The title reagent is used as a nucleophilic fluorinating agent.

Kvícala, J.; Mysík, P.; Paleta, O. Synlett 2001, 545.

$$
\begin{aligned}
& \mathrm{Me}, \mathrm{F}^{\mathrm{i}}-\mathrm{Bu}_{4} \mathrm{~N}^{+} \\
& \mathrm{Me}-\underset{\mathrm{F}}{\mathrm{i}-\mathrm{Bu}}
\end{aligned}
$$

A
26 examples (yields 85-94\%).

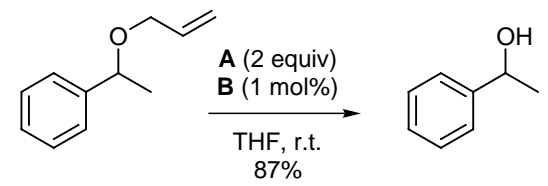

